\title{
Philosophiques
}

\section{Gilles-Gaston Granger, La vérification, Paris, Éditions Odile Jacolo, 1992, 314 pages.}

\section{Yvon Gauthier}

Volume 20, numéro 2, automne 1993

Perspectives sur la phénoménologie et l'intentionnalité

URI : https://id.erudit.org/iderudit/027243ar

DOI : https://doi.org/10.7202/027243ar

Aller au sommaire du numéro

Éditeur(s)

Société de philosophie du Québec

ISSN

0316-2923 (imprimé)

1492-1391 (numérique)

Découvrir la revue

Citer ce compte rendu

Gauthier, Y. (1993). Compte rendu de [Gilles-Gaston Granger, La vérification, Paris, Éditions Odile Jacob, 1992, 314 pages.] Philosophiques, 20(2), 514-516.

https://doi.org/10.7202/027243ar

Ce document est protégé par la loi sur le droit d'auteur. L'utilisation des services d'Érudit (y compris la reproduction) est assujettie à sa politique d'utilisation que vous pouvez consulter en ligne.

https://apropos.erudit.org/fr/usagers/politique-dutilisation/ 
Gilles-Gaston Granger, La vérification, Paris, Éditions Odile Jacob, 1992, 314 pages.

\section{par Yvon Gauthier}

D'entrée de jeu, le dernier ouvrage de G. G. Granger se présente comme un essai d'épistémologie comparative, c'est-à-dire un ensemble plus ou moins unifié d'analyses autour d'un thème, ici celui de la vérification. Les trois parties de l'ouvrage ( Connaissance symbolique, connaissance intuitive et vérité », «Vérifier en mathématiques » et "Vérité et expérience ») comportent une grande variété d'exemples ou de cas exemplaires, par exemple le nominalisme occamiste, la théorie nominaliste de la physique de $\mathrm{H}$. Field, la théorie wittgensteinienne du Tractatus, celle de Carnap dans son Logischer Aufbau der Welt, la théorie tarskienne de la vérité, la théorie restreinte de la relativité, la mécanique quantique, la logique intuitionniste, la logique quantique, la théorie de l'utilité espérée en économique, le théorème des quatre couleurs (Appel-Haken), les théories de Sneed et Scheibe en philosophie des sciences, etc. Cette énumération est partielle et la prolifération des exemples est si grande que l'on peut à juste titre parler d'analyses ponctuelles dans la plupart des cas. Une telle richesse ne serait pas surenchère, si la thèse comparatiste ne se résumait pas à une théorie générale de la distinction forme / contenu ou opératoire / objectal (p. 3or). Le relativisme modéré (p. Io) de cette épistémologie mitigée nous renseigne peu à la limite sur la vérification dans les théories scientifiques, malgrè la grande clarté des analyses et l'information historique qu'elles véhiculent - on pourra consulter pour le débat contemporain La nature de la vérité scientifique (CIACO, Louvain-la-Neuve, I985).

Je veux relever quelques exemples d'un traitement comparatif qui marque les limites d'une analyse de cas. Prenons le cas de la logique : l'A. nous dit que seule la logique classique du vrai et du faux pour les énoncés (p. I3i) serait la 
logique pure, alors que la logique intuitionniste (p. 165) et la logique quantique (p. 216), comme la logique des prédicats du $\mathrm{I}^{\mathrm{er}}$ ordre seraient des logiques «à contenus ». Rappelons que Brouwer lui-même a admis la possibilité d'une logique mathématique intuitionniste pour les énoncés négatifs et que la réinterprétation intuitionniste des constantes logiques n'en fait pas moins une logique bivalente avec valeurs dans $(0, \mathrm{I})$ tout comme l'algèbre booléenne partielle des événements quantiques dans le résultat de Kochen-Specker - qui est une généralisation du résultat de von Neumann-Birkhoff discuté par l'A. (p. 215-217). Le résultat de Kochen-Specker suffit à invalider la thèse de l'A. qui voudrait que seule " la structure booléenne qui fournit un "modèle" à la logique des énoncés est purement opératoire » ou encore qu'elle soit un «système opératoire minimal permettant d'opérer sur des objets en général » (p. I67). Or le théorème de Kochen-Specker montre justement que la logique quantique est une algèbre de Boole partielle qui n'est pas plongeable dans une algèbre de Boole - il n'y a pas d'homomorphismes bivalués de celle-là dans celle-ci. Il est donc manifeste que la logique classique n'est qu'une logique interne, «à contenus », des situations symétriques finies. Les logiciens conviennent depuis longtemps que le domaine de la logique du i $\mathrm{I}^{\mathrm{er}}$ ordre est défini par les thëorèmes de complétude, Löwenheim-Skolem et compacité (théorème de Lindström) et il est vain de chercher dans la structure algébrique quelque fondement dernier du formel ou de l'opératoire. l'imprécision de la thèse conduit parfois à des confusions élémentaires sur le paradoxe de Skolem (p. 134 et 142) dont on nous dit qu'il se résume à la formule « toute théorie ou toute proposition qui a un modèle infini a nécessairement un modèle infini dénombrable ». Il faudrait ajouter ici «du Ierordre » et distinguer le paradoxe du théorème de Löwenheim-Skolem ascendant (dû à Tarski) qui nous dit que toute théorie du $\mathrm{I}^{\mathrm{er}}$ ordre qui a un modèle infini dénombrable en a un de cardinalité infinie arbitraire; le paradoxe consiste à parler d'un infini non dénombrable à l'intérieur d'une théorie dénombrable et sa résolution tient simplement à ce que la bijection entre $\aleph_{0}$ et $2 \aleph^{\circ}$ ou l'ensemble des parties de $\aleph_{0}$ n'est pas dans le modèle de la théorie, mais dans l'univers $\mathrm{V}$ des ensembles. Confusion aussi sur le premier théorème d'incomplétude de Gödel (p. II8), comme si ce dernier requérait la théorie axiomatique des ensembles de Zermelo-Fraenkel dans sa formulation. Peut-être est-ce là l'effet d'une logique «à contenus »? La thèse simplifie à outrance le problème de la vérification en théorie des nombres, pour donner un autre exemple. Il faudrait faire ici les distinctions utiles entre théorie constructive et théorie analytique, théorèmes d'existence et méthodes effectives qui sont des vérifications au-delà de la preuve transcendante pour contredire un constat comme le suivant : « c'est ainsi qu'en théorie des nombres la "vérification" n'est jamais qu'un appel à la démonstration » (p. g8).

Prenons un autre exemple : la théorie de H. Field dans Science without Numbers (Princeton University Press, 1980). La théorie de Field repose sur la notion d'extension conservatrice d'une théorie du premier ordre et vise à réduire le contenu mathématique d'une théorie physique comme la gravitation universelle de Newton au statut d'extension conservatrice. L'A. insiste (p. 274 sq.) sur le caractère « conservateur » de la théorie de Field, sans noter son « extensibilité », pourrait-on dire. Il importe, en effet, de reconnaitre que seule 
une théorie du r rerdre peut s'adjoindre ainsi des constantes, par exemple, pour changer le corps de théorèmes de la théorie. Quoi que vaille ultimement ce nominalisme radical, c'est sur son pouvoir d'extension ou d'absorption qu'il faut l'évaluer et non sur ses vertus « conservatrices ». Venant à la théorie sneedienne - l'A. s'inspire ici de l'excellent ouvrage La sémantique des théories physiques (Presses de l'Université d'Ottawa, 1988) de Jean Leroux - on nous dit qu'elle « établit l'incomplète réductibilité du théorique ( (p. 272). Enfin, pour la théorie de Scheibe-Ludwig (p. 278 sq.) sur l'interprétation bourbakiste des théories, il serait intéressant de se demander comment la prétendue catégoricité de la structure d'une théorie physique peut échapper à la critique de Putnam (« Models and Reality ») (J.S.L.) qui montre qu'une théorie physique formulée au premier ordre ne peut être catégorique en vertu du théorème de Löwenheim-Skolem. La question n'est pas posée par l'A.

Une autre question pourrait être adressée au relativisme modéré de l'A. Comment réconcilier une interprétation du paradoxe d'Einstein-Podolsky-Rosen (p. Ig4 sq.) en termes de variables cachées comme « faits virtuels » avec une interprétation probabiliste des inégalités de Bell ou encore une interprétation statistique des résultats de mesure en mécanique quantique? I'A. invoque les notions de virtualité interne et de virtualité externe (p. 218-219) pour combler cet écart entre probabilités et statistiques, mais un réaliste modéré pas plus qu'un nominaliste modéré (encore moins un constructiviste radical) ne sauraient s'en satisfaire. Il faudrait sans doute poser la question en termes de probabilités pseudo-booléennes et d'une logique locale non commutative, ce que ne fait pas l'A.

Les remarques précédentes témoignent de la richesse des analyses et de l'ampleur des questions soulevées. La variété étale des exemples et le manque de relief dans le traitement des cas spécifiques atténuent cependant une thèse inspirée par la modération épistémique et par la recherche pondérée d'une « corrélation d'objets de pensée et de systèmes opératoires » (p. 30I).

Département de philosophie

Université de Montréal 